\title{
Revista Chilena de
}

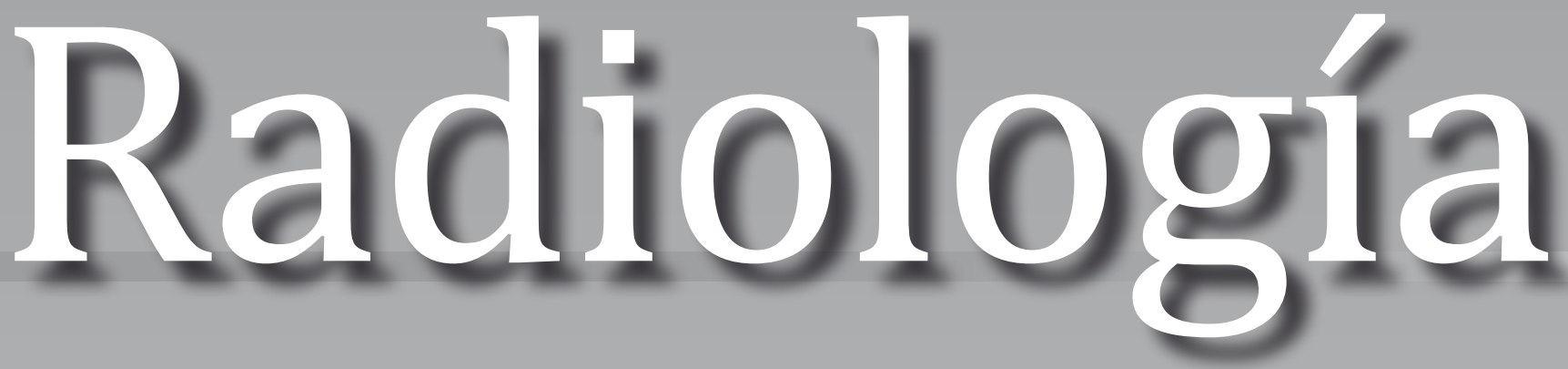

\section{ÓRGANO OFICIAL DE LA SOCIEDAD CHILENA DE RADIOLOGÍA}

\section{EDITOR CIENTÍFICO}

DR. CLAUDIO SILVA F-A.

Clínica Alemana de Santiago. Chile.

\section{COMITÉ EDITORIAL}

DR. CLAUDIO CORTÉS A.

Hospital Clínico Universidad de Chile,

Clínica Alemana de Santiago. Chile.

DR. MARCELO GALVEZ M. (Coeditor)

Clínica Las Condes. Chile.

DR. ANDRÉS O'BRIEN S. (Coeditor)

Clínica Las Condes. Chile.

DR. GIANCARLO SCHIAPPACASSE F.

Clínica Alemana de Santiago. Chile.

Dirección: General Holley 2363-A, Of. 404. Providencia, Santiago de Chile.

Secretaria de la Sociedad de Radiología

Fono: 3789739 - Fax: 2319103

http://www.sochradi.cl

e-mail:contacto@sochradi.cl

La Revista es distribuida en forma gratuita a todos los miembros al día de la Sociedad Chilena de Radiología y Medicina Nuclear.

\author{
DIRECTORIO SOCIEDAD \\ CHILENA DE RADIOLOGÍA A.G \\ $2011-2013$ \\ PRESIDENTE \\ Dr. Miguel Ángel Pinochet T. \\ VICE-PRESIDENTE \\ Dr. Pablo Soffia S. \\ SECRETARIA GENERAL \\ Dra. Marcela Cortés S. \\ TESORERA \\ Dra. Isabel Fuentealba T. \\ DIRECTORA GREMIAL \\ Dra. Teresa Taub E. \\ DIRECTORA DE PUBLICACIONES \\ Dra. Patricia Orellana P. \\ SECRETARIA DE ACTAS \\ Dra. Georgette Pose L. \\ PAST-PRESIDENT \\ Dra. Gloria Soto G. \\ DIRECTOR ZONAL REGIONES XV, I y II \\ Dr. Paulo Flores $\mathrm{G}$. \\ DIRECTOR ZONAL REGIONES III Y IV \\ Dra. María Loreto Vergara del $R$. \\ DIRECTOR ZONAL REGIÓN V \\ Dra. Patricia Manquez $\mathrm{H}$. \\ DIRECTOR ZONAL REGIONES VI y VII \\ Dr. Víctor Manuel Retamal P.
}

DIRECTOR ZONAL REGIONES VIII, IX, X y XIV Dr. Claudio Medina G.

La periodicidad de la Revista Chilena de Radiología es trimestral y el contenido de cada artículo y/o anuncio es responsabilidad del autor, sin que necesariamente represente los puntos de vista de los editores. La reproducción total o parcial sólo puede hacerse con previa autorización escrita de Publimpacto. Esta revista está indizada a la base de datos referenciales LILACS y SciELO.

En su versión electrónica en el sitio WwW.scielo.cl se encuentran todos sus artículos en forma completa.

Revista Chilena de Radiología es una producción de Publimpacto

Robinson Crusoe 1150 Of. 904, Las Condes. Santiago de Chile. Cód. Postal: 7570668 Fono: (56-2) 7-8619516 • 9-5094865

Editor Comercial: Sr. Patricio Gana G. • eganag@gmail.com

Representante venta de publicidad: Sra. Paulina Gana・pganag@gmail.com 


\section{ÍNDICE}

EDITORIAL

- Investigación en radiología y la formación del residente

Claudio Silva F-A

\section{CASO DESAFÍO DIAGNÓSTICO}

- Dolor perineal y disfunción sexual

Nicolás López G, Alberto Marangoni, Tania Buccolini, Marco Marangoni,

Rodrigo Re, Jorge Galíndez, Juan Gagliardino, Daniel Benítez.

\section{ARTICULOS ORIGINALES}

GASTROINTESTINAL

- Estudio baritado del tubo digestivo alto en pacientes postoperados de cirugía bariátrica: Hallazgos normales y patológicos

José A. de Grazia K, Marcelo Godoy Z, Ignacia Torrealba A, Mauricio Guzmán G, Paula Csendes G, Attila Csendes J.

NEURORRADIOLOGÍA

- Trombectomía Mecánica en el ICTUS; Experiencia con Trevo en Hospital Provincial Clínico Barcelona

Daniel Campodónico O, Antonio López R, Luis San Román M, Jordi Blasco A, Laura Oleaga Z, Juan Macho F.

\section{CARDIOTORÁCICO}

- Correlación entre score de calcio coronario, esteatosis hepática y síndrome metabólico

Karina Hermosilla M, Daniela Pivcevic C, Julia Alegría B, Claudio Silva F.

\section{ARTICULOS DE REVISIÓN}

MUSCULOESQUELÉTICO

- Osteocondroma: diagnóstico radiológico, complicaciones y variantes

Marco Cañete P, Elena Fontoira M, Begoña Gutiérrez San José, Slavina Mancheva $M$.

SIGNOS RADIOLÓGICOS

- Signo Radiológico: "El lirio caído"

Samuel Sánchez C, M. Carolina Pérez S.

\section{COMUNICACIONES}

- RESULTADO CASO DESAFÍO DIAGNÓSTICO 


\title{
EDITORIAL
}

\section{Investigación en radiología y la formación del residente}

Los programas de formación de la especialidad en radiología contemplan al menos tres años de un intenso proceso orientado a adquirir competencias y habilidades para el correcto desempeño de la profesión, incorporar nociones avanzadas de protección radiológica, calidad, y adecuada integración de los procedimientos, todo bajo la atenta supervisión y apoyo de Médicos Radiólogos calificados e involucrados en este proceso docente. En los últimos años ha sido una tendencia mundial en los programas de formación de nuestra especialidad, la incorporación de competencias y habilidades referido a temas de investigación y/o metodología de radiología basada en evidencias. Ello busca inculcar conceptos operacionales claros, estos son (entre otros): identificar los vacíos del conocimiento, lo cual involucra necesariamente habilidades para poder localizar en el mar de información disponible aquellos que sea pertinente a la duda en cuestión. Una vez identificado este vacío del conocimiento, el poder disponer de herramientas que permitan formular un método adecuado para poder responder aquella inquietud de una forma segura para el paciente, que sea metodológicamente plausible y además, éticamente ejecutable. Es en este proceso de pensamiento activo y crítico, que el residente va adquiriendo aquellas habilidades que le permitirán, entre otras cosas, lograr un nivel de comprensión y fomentar su autoaprendizaje continuo. Además, el involucrarse efectivamente en un proyecto de investigación implica desarrollar elementos de disciplina y orden que trae por añadiduría una comprensión cabal de la temática que se está estudiando.

Esto solamente lleva a un círculo virtuoso del conocimiento, generación de conocimiento, y difusión de éste a la comunidad. Son los residentes del presente que van a poder crear y avanzar en el conocimiento de nuestra especialidad al futuro y serán los que darán motor y energía para mantener esta especialidad activa y vigente como lo es hasta ahora. Es por ello que se hace absolutamente necesario proveer del apoyo tanto técnico como metodológico y docente, a los residentes para que puedan adquirir estas habilidades, proveerles de tiempo protegido y de facilidades para poder generar sus trabajos y también poder difundir los resultados de ellos.

El Congreso anual de Radiología organizado por la Sociedad Chilena de Radiología es una excelente oportunidad para poder dar ese empuje inicial a aquellos estudios que estén generando y que estén apoyados por docentes calificados y así difundir la labor que se realiza dentro de las instituciones académicas. Ello permitirá lograr una legión creciente de radiólogos capaces e interesados en el crecimiento y desarrollo de nuestra especialidad. Las condiciones están, el material humano está, sólo se requiere dar el paso que han tomado instituciones académicas por todo el mundo y ojalá encontrar una vía de coordinación local para facilitar estos procesos.

\author{
Dr. Claudio Silva F-A \\ Editor Científico
}

